\title{
Role of biofilms in fungal keratitis- an overview
}

\begin{abstract}
Biofilms are generally defined as a community of sessile microbes held together by a polymeric extracellular matrix (ECM), adherent to a surface that are phenotypic ally distinct from their plank tonic counterparts. Fungal keratitis is an important cause of ocular morbidity in the form of corneal opacity and visual loss, especially in developing countries Predisposing factors for fungal keratitis include tropical or subtropical climate, agricultural work, contact lens users and corneal trauma. Fungal keratitis is most commonly caused by filamentous fungi like Fusarium spp. (F. solani and F. oxysporum) followed by Aspergillus spp. and dematiaceous fungi. Fungal biofilm formation plays an important role in the development of fungal keratitis. Here in this review, we will be discussing the formation of biofilms and its role in fungal keratitis.
\end{abstract}

Volume 5 Issue 6 - 2017

\author{
Shweta Sharma, Sudhir Singh, Shewtank Goel, \\ Umar Farooq \\ Department of Microbiology, Teerthankar Mahaveer Medical \\ College and Research Centre, India
}

\begin{abstract}
Correspondence: Sharma S, Department of Microbiology, Teerthankar Mahaveer Medical College and Research Centre, Moradabad, India, Email drshwetamicro@gmail.com
\end{abstract}

Received: August 26, 2017| Published: December 05, 2017

Keywords: biofilm, fungal keratitis, contact lens

\section{Introduction}

Biofilm formation has been detected quite early in the fossil record (approx. 3.25billionyears ago and is common throughout diverge range of organisms. However, the first description of a biofilm dates back to the $17^{\text {th }}$ century when Anthony van Leeuwenhoek noted the presence of small living "animalcules" in his dental plaque. ${ }^{1}$ But nothing was clear and it took an additional 200years until the germ theory of disease was advanced by Robert Koch before a connection between microbes and disease was made. ${ }^{2}$ Recent advances in confocal microscopy and molecular genetics have provided evidence that biofilm formation is the most common mode of growth of microorganisms in nature. This growth form allows microbial cells to survive in hostile environments, enhances their resistance to physical and chemical pressures, and promotes metabolic cooperation. ${ }^{2}$ In fact, it is estimated that approximately $80 \%$ of all bacteria in the environment exist in biofilm communities, and more than $65 \%$ of human microbial infections involve biofilms. ${ }^{3}$ Microbial biofilms are communities of micro-organisms that are strongly attached to biologic and nonbiologic substrata, enclosed in a self-produced protective exopolymeric matrix (EPM). It is seen mixed polymicrobial biofilms are common and can be considered even more complex communities of microbes that cooperatively interact in an altruistic manner. ${ }^{4}$ The type of organisms causing keratitis depends on geographical conditions such as differences in climate, environment and occupational risk. ${ }^{5}$ Bacterial keratitis, especially contact lensassociated infection, is caused by both Gram negative pathogens, such as $P$. aeruginosa and Serratia spp., and Gram positive organisms, such as $S$. aureus $^{6}$ and other staphylococcal species. ${ }^{5,7}$ The recognition of fungal biofilms brought attention to nosocomial infections and now it is believed that several fungal genera are capable of organizing into a biofilm. ${ }^{8}$ Here in this review, we will be discussing the formation of fungal biofilms and its role in fungal keratitis.

\section{Fungal biofilms}

Biofilms are generally defined as a community of sessile microbes (bacterial or fungal) held together by a polymeric extracellular matrix (ECM), adherent to a surface that are phenotypically distinct from their planktonic counterparts. . . Members of a biofilm community, which can be of the same or multiple species, show varying stages of differentiation and exchange information, metabolites, and genes with each other. As a result, members of the biofilm community are in a diversity of physiologies influenced by the unequal sharing of nutrients and metabolic byproducts, which results in subpopulations with increased tolerance to anti fungals and environmental stresses, the host immune system, and predatory microorganisms. ${ }^{3,10-12}$ The process of biofilm formation occurs in five stages (1) reversible aggregation of planktonic cells on a surface; (2) irreversible adhesion; (3) formation of microcolonies; (4) biofilm maturation; and (5) detachment and dispersion of cells. ${ }^{10,13}$ Candida albicans biofilms are comprised primarily of yeast-form and hyphal cells, both of which are required for biofilm formation..$^{14}$ Formation is a sequential process involving adherence to a substrate (either abiotic or mucosal surface), the proliferation of yeast cells over the surface, and induction of hyphal formation..$^{14}$ ECM accumulates as the biofilm matures, and seems to contribute to cohesion. ${ }^{15}$ C. albicans biofilms form on numerous abiotic and biotic surfaces. ${ }^{16,17}$ Other Candida spp. including $C$. tropicalis, C. parapsilosis, and C. glabrata form ECM-containing biofilms but do not produce true hyphae. ${ }^{18}$ Aspergillus biofilms can form both on abiotic and biotic surfaces. ${ }^{19}$ The initial colonizing cells that adhere to the substrate are conidia. Mycelia (the hyphal form) develop as the biofilm matures. ${ }^{19}$ Hyphae of $C$. albicans and of $A$. fumigatus can form pores or channels through biotic surfaces. ${ }^{20,21}$ Other fungal pathogens Trichosporon asahii, Coccidioidesimmitis, C. neoformans, Pneumocystis species etc. do not produce hyphal structures as part of their biofilms (Figure 1). ${ }^{22}$

\section{Fungal keratitis}

Fungal keratitis is an important cause of ocular morbidity in the form of corneal opacity and visual loss especially in developing countries, where it may account for nearly half of corneal ulcers. ${ }^{23}$ Predisposing factors for fungal keratitis include tropical or subtropical climate, agricultural work, contact lens users and corneal trauma. Fungal keratitis is most commonly caused by filamentous fungi like Fusarium spp. (F. solani and F. oxysporonoxysporum) followed by Aspergillus spp. and dematiaceous fungi (Figure 2). ${ }^{23,24}$ 




Figure I Steps of biofilm formation.

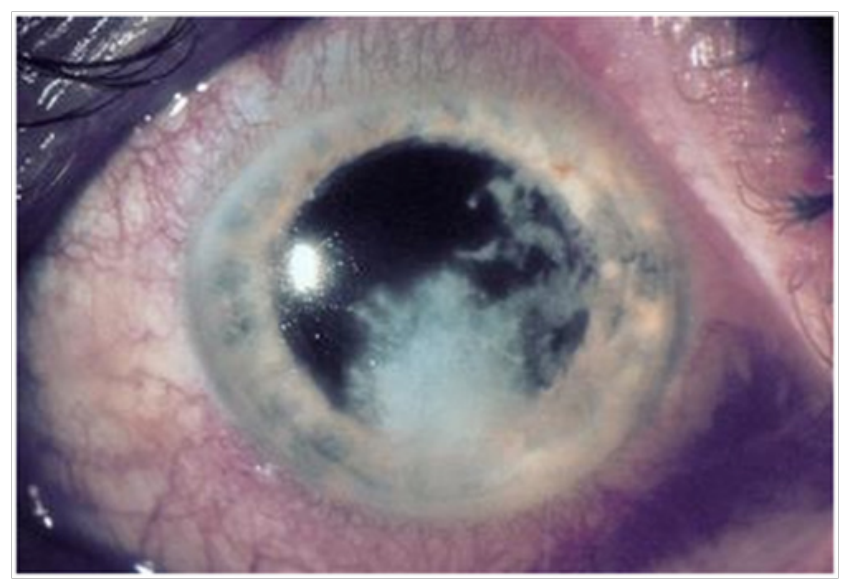

Figure 2 Fungal keratitis caused by filamentous fungi (lesion showing feathery edges).

\section{Contact lens-associated fungal keratitis}

High risk of fungal keratitis in contact lens wearers has been associated with the ability of the lens to induce modification of the corneal epithelium and to carry organisms to the ocular surface. ${ }^{25,26}$ Use of lens induces local alterations like hypoxia and hypercapnia, which affect the ability of the epithelium to respond to damage. Tear fluid exchange may also be compromised between the anterior and posterior sides of the lens thereby limiting its antimicrobial properties. ${ }^{25}$ In addition, contact lenses provide a surface where microorganisms may attach and colonize the surface as a biofilm and can spread to a previously damaged corneal epithelium. ${ }^{26}$ Poor hygiene and infrequent replacement of the contact lens storage cases are independent risk factors for moderate and sever keratitis. ${ }^{27}$ The incidence of contact lens-associated microbial keratitis has been shown to be impacted by the contact lens material, and also by the wear schedule. Higher risk for development of keratitis was seen with daily wear soft contact lenses compared to daily wear rigid gas permeable lenses, and it was further increased for extended wear (overnight wear) soft contact lenses. ${ }^{28,29}$ Recently introduced daily disposable and silicone hydrogel contact lenses have also been associated with higher incidence of keratitis compared to rigid gas permeable contact lenses..$^{30,31}$

\section{Role of contact lenses in biofilm formation}

Once the lens is placed on the surface of the eye, the disinfectant diffuses off the lens and is replaced by lipids and proteins present in the tear fluid. ${ }^{32}$ Microbial adhesion to surfaces coated by with proteins and other biomolecules is often accomplished by a class of molecules termed Microbial Surface Components Recognizing Adhesive Matrix Molecules (MSCRAMM), as well as other adhesive surface proteins. ${ }^{33}$ In a moving suspension, cells are exposed to fairly uniform conditions. However, following attachment, the individual experience of a cell begins to differ from its neighbors (i.e., a cell in the middle of a group will experience more excreted products and fewer factors from the environment than a cell on the periphery of a population), and as a result, cells begin to differentiate. ${ }^{34}$ Many biofilms involve the production of an extracellular matrix (ECM) that encases the cells, and in some cases, binds the cells together and that can be composed of polysaccharides, lipopolysaccharides, proteins, or extracellular DNA..$^{34}$ Extracellular matrix contributes to the differentiation of cells within the encased population and protects the organism from the action of antimicrobial agents, host immune responses, bacteriophages and phagocytic amoeba. ${ }^{35}$

\section{Role of biofilms in fungal keratitis}

Keratitis caused by Fusarium $F$. solani and F. oxysporum is among the most refractory and common causes of fungal keratitis. Fusarium is a filamentous fungus that infests agricultural plants, and the incidence of Fusarium keratitis peaks during harvest seasons when farm workers at are more risk of corneal injury and exposure to airborne spores. ${ }^{35}$ However, another major risk factor for Fusarium keratitis is contact lens wear due to increased association with contaminated lenses, lens cases, and contact lens care solutions. The formation of biofilm by these fungi on contact lenses and lens cases is thought to have a major role in causing keratitis once the lens is placed on the ocular surface. ${ }^{36}$ From 2005-2007, more than 300 cases of Fusarium keratitis were associated with contact lens wear and in some cases, characterized as a serious disease in which patient underwent corneal transplantation or even complete eye removal. The severity of disease was assigned to misdiagnosis and inappropriate treatment, and to the failure of antimycotic agents due to the resistance exhibited by the micro-organisms in the biofilms. ${ }^{37}$ Microbial biofilms have been 
observed on contact lenses and the 2005-2006 outbreak was attributed partly to the ability of Fusarium to form biofilms. ${ }^{38,39}$ Another study showed that Fusarium isolates from the outbreak formed biofilms on soft contact lenses in vitro and in vivo tested inamurine model, and that biofilm formation was elevated in $F$. solani compared to $F$. oxysporum isolates. These biofilms were susceptible to natamycin, but exhibited species-dependent susceptibility to Amphotericin B and Voriconazole. $^{40,41}$

\section{Conclusion}

The Ability of Fungi fungi to form biofilms is a critical determinant in resistance to anti fungals and to the pathogenesis of fungal keratitis, and it should be taken into consideration when managing patients with this disease. However, further research is required in the field of fungal biofilms and their role in human diseases.

\section{Acknowledgements}

None.

\section{Conflict of interest}

The author declares no conflict of interest.

\section{References}

1. Van Leeuwenhoek A. Microscopical observations about animals in the scurf of the teeth. Philos Trans. 1684;14:568-574.

2. Jabra-Rizk MA, Falkler WA, Meiller TF. Fungal biofilms and drug resistance. Emerg Infect Dis. 2004;10(1):14-19.

3. Donlan RM, Costerton JW. Biofilms: Survival mechanisms of clinically relevant microorganisms. Clin Microbiol Rev. 2002;15(2):167-193.

4. Hall-Stoodley L, Costerton JW, Stoodley P. Bacterial biofilms: from the natural environment to infectious diseases. Nat Rev Microbiol. 2004;2(2):95-108.

5. Shah A, Sachdev A, Coggon D, et al. Geographic variations in microbial keratitis: An analysis of the peer-reviewed literature. Br J Ophthalmol. 2011;95(6):762-767.

6. Alexandrakis G, Alfonso EC, Miller D. Shifting trends in bacterial keratitis in South Florida and emerging resistance to fluoroquinolones. Ophthalmology. 2000;107(8):1497-1502

7. Lichtinger A, Yeung SN, Kim P, et al. Shifting trends in bacterial keratitis in toronto: An 11-year review. Ophthalmology. 2012;119(9):1785-1790.

8. Sardi JDCO, Pitangui NDS, Rodríguez-Arellanes G, et al. Highlights in pathogenic fungal biofilms. Rev Iberoam Micol. 2014;31(1):22-29.

9. Mukherjee PK, Chandra J. Candida biofilm resistance. Drug Resist Update. 2004;7(4-5):301-309.

10. Donlan RM, Costerton JW. Biofilms:Survival mechanisms of clinically relevant microorganisms. Clin Microbiol Rev. 2002;15(2):167-193.

11. Annous BA, Fratamico PM, Smith JL. Scientific status summary. J Food Sci. 2009;74(1):R24-R37.

12. Hooshangi S, Bentley WE. From unicellular properties to multicellular behavior: Bacteria quorum sensing circuitry and applications. Curr Opin Biotechnol. 2008;19(6):550-555

13. Stewart PS, Franklin MJ. Physiological heterogeneity in biofilms. Nat Rev Microbiol. 2008;6(3):199-210.

14. Behlau I, Gilmore MS. Microbial biofilms in ophthalmology and infectious disease. Arch Ophthalmol. 2008;126(11):1572-1581.

15. Finkel JS, Mitchell AP. Genetic control of Candida albicans biofilm development. Nat Rev Microbiol. 2011;9(2):109-118.
16. Al-Fattani MA, Douglas LJ. Biofilm matrix of Candida albicans and Candida tropicalis: chemical composition and role in drug resistance. $J$ Med Microbiol. 2006;55(Pt 8):999-1008.

17. Dongari-Bagtzoglou A, Kashleva H, Dwivedi P, et al. Characterization of mucosal Candida albicans biofilms. PLoS One. 2009;4(11):e7967.

18. Harriott MM, Lilly EA, Rodriguez TE, et al. Candida albicans forms biofilms on the vaginal mucosa. Microbiology. 2010;156(Pt 12):36353644.

19. Silva S, Negri M, Henriques M, et al. Adherence and biofilm formation of non-Candida albicans Candida species. Trends Microbiol. 2011;19(5):241-247.

20. Mowat E, Williams C, Jones B, et al. The characteristics of Aspergillus fumigatusmycetoma development: is this a biofilm? Med Mycol. 2009;47(1):S120-S126.

21. Singhal D, Baker L, Wormald PJ, et al. Aspergillus fumigatus biofilm on primary human sinonasal epithelial culture. Am J Rhinol Allergy. 2011;25(4):219-225.

22. Lermann U, Morschhauser J. Secreted aspartic proteases are not required for invasion of reconstituted human epithelia by Candida albicans. Microbiology. 2008;154(Pt 11):3281-3295.

23. Cushion MT, Collins MS, Linke MJ. Biofilm formation by Pneumocystis spp. Eukaryot Cell. 2009;8(2):197-206.

24. Thomas PA. Current perspectives on ophthalmic mycoses. Clin Microbiol Rev. 2003;16(4):730-797.

25. Hu SBS, Fan VC, Koonapareddy C, et al. Contact lens-related Fusarium infection: case series experience in New York City and review of fungal keratitis. Eye Contact Lens. 2007;33(6 pt 1):322-328.

26. Fleiszig SM, Evans DJ. Pathogenesis of contact lens-associated microbial keratitis. Optom Vis Sci. 2010;87(4):225-232.

27. Willcox MD, Carnt N, Diec J, et al. Contact lens case contamination during daily wear of silicone hydrogels. Optom Vis Sci. 2010;87(7):456464 .

28. Stapleton F, Edwards K, Keay L, et al. Risk factors for moderate and severe microbial keratitis in daily wear contact lens users. Ophthalmology. 2012;119(8):1516-1521.

29. Cheng $\mathrm{KH}$, Leung SL, Hoekman HW, et al. Incidence of contactlens-associated microbial keratitis and its related morbidity. Lancet. 1999;354(9174):181-185.

30. Dart JK, Stapleton F, Minassian D. Contact lenses and other risk factors in microbial keratitis. Lancet. 1991;338(8768):650-653.

31. Dart JK, Radford CF, Minassian D, et al. Risk factors for microbial keratitis with contemporary contact lenses: A case-control study. Ophthalmology. 2008;115(10):1647-1654.

32. Stapleton F, Keay L, Edwards K, et al. The incidence of contact lens-related microbial keratitis in australia. Ophthalmology. 2008;115(10):1655-1662.

33. Bright FV, Merchea MM, Kraut ND, et al. A preservative-and-fluorescein interaction model for benign multipurpose solution-associated transient corneal hyperfluorescence. Cornea. 2012;31(12):1480-1488.

34. Patti JM, Allen BL, McGavin MJ, et al. Mscramm-mediated adherence of microorganisms to host tissues. Annu Rev Microbiol. 1994;48:585617.

35. Flemming HC, Wingender J. The biofilm matrix. Nat Rev Microbiol. 2010;8(9):623-633.

36. Bharathi MJ, Ramakrishnan R, Meenakshi R, et al. Microbial keratitis in South India: influence of risk factors, climate, and geographical variation. Ophthalmic Epidemiol. 2007;14(2):61-69. 
37. Imamura Y, Chandra J, Mukherjee PK, et al. Fusariumand Candida albicans biofilms on soft contact lenses: model development, influence of lens type and susceptibility to lens care solutions. Antimicrob Agents Chemother. 2008;52(1):171-182.

38. Donnio A, Van Nuoi DN, Catanese M, et al. Outbreak of keratomycosis attributable to Fusariumsolani in the French West Indies. Amer $J$ Ophthalmol. 2007;143(2):356-358.

39. Dyavaiah M, Ramani R, Chu D. Molecular characterization, biofilm analysis and experimental biofouling study of Fusarium isolates from recent cases of fungal keratitis in New York State. BMC Ophthalmology. 2007;7:1.
40. Behlau I, Gilmore MS. Microbial biofilms in ophthalmology and infectious disease. Arch Ophthalmol. 2008;126(11):1572-1581.

41. Mukherjee PK, Chandra J, Yu C. Characterization offusarium keratitis outbreak isolates: Contribution of biofilms to antimicrobial resistance and pathogenesis. Investig Ophthalmol Vis Sci. 2012;53:4450-4457. 